\title{
MODEL CALCULATIONS OF CONTINUOUS GRAIN GROWTH IN TEXTURED MATERIALS
}

\author{
H. J. BUNGE and E. DAHLEM-KLEIN \\ Department of Physical Metallurgy, Technical University Clausthal, Germany
}

(Received 18 June 1990)

\begin{abstract}
Continuous grain growth of a polycrystalline structure proceeds by the simultaneous movement of all grain boundaries driven by the grain boundary energy and controlled by the boundary mobility. In the present model calculations, energy and mobility are allowed to depend on the orientation difference between neighbouring crystals. The growth process then depends on the misorientation distribution function. In a first approximation the orientation correlations are assumed to be random. Then the MODF can be expressed by the texture. A simple growth model leads to a continuity equation depending on crystal orientation $g$ and grain radius $r$ which can be integrated for the steady state case in which the texture and the shape of the grain size distribution curve remain constant. The same solution also serves as an approximation for pseudo-steady state cases with slowly varying texture. More general solutions are obtained by adding several pseudo-steady state solutions. The specific case of two pseudo-steady state components is considered in detail. Thereby the growth kinetics, texture changes and the form of the grain size distribution curves is obtained.
\end{abstract}

KEY WORDS Continuous grain growth, continuity equation, analytical steady state solution, pseudo-steady state solutions, one and two component case

\section{INTRODUCTION}

During annealing the grain size of a polycrystalline structure may continuously increase, driven by a reduction of grain boundary energy. The driving force of this recrystallization process is much smaller than that of primary recrystallization. Hence, continuous grain growth may strongly depend on all kinds of additional driving or impeding forces of the same order of magnitude such as dislocation substructures, internal stresses, solute atoms especially concentrated in grain boundaries, and second phase particles. If one considers an ideally pure and homogeneous material, these effects may be assumed to be small compared with grain boundary energy. Continuous grain growth is then controlled solely by grain boundary energy and mobility. In an ideal material of this type the specific grain boundary energy as well as the mobility must be assumed to be constant throughout the process. Both quantities will, however, strongly depend on the orientation difference across the grain boundaries and the orientation of the boundary itself, i.e. on five grain boundary orientation parameters.

In detail, the movement of any grain boundary point must be assumed to be determined by the local curvature, the local mobility and the local specific energy. These quantities, in turn, are controlled by the topological necessity for the grain boundaries to form a connected three-dimensional structure, and by the crystal orientations of neighbouring grains. Starting with a given grain structure and with given grain orientations the growth process can then be followed step by step by 
computer simulation following the movement of each point of each grain boundary throughout the whole growth process. This is the most direct way to model continuous grain growth (see e.g. Anderson, 1986, Grest, Anderson and Srolowitz 1986). Model calculations of this type are theoretically very satisfactory in as far as they are based on "first principles." They are, however, at the same time also very time consuming if all relevant structural parameters are to be included in the calculations. Furthermore, they have the disadvantage that the results, strictly speaking, apply only to the particular structure chosen for the initial state. In order to generalize the results to a whole "class" of structures, the calculations have then to be repeated for a number of individual grain structures which are considered to be representative for what is called the "class" of grain structures. In fact, this latter uncertainty detracts considerably from the theoretical advantage of starting from first principles. The definition of a "statistically relevant class" of grain structures is often not very clear.

Another group of model calculations, so to speak, inverts the sequence of assumptions which are being made. At first one decides which parameters of a polycrystalline structure are assumed to be "statistically relevant" and then one assumes a growth model which can be formulated within these assumed parameters. This type of model calculation was first used by Hillert (1965). He assumed the grain radius $r$ to be the controlling variable. In a more detailed model the radius $r$ and number of faces of a grain can be considered as separate variables (Kurtz and Carpay 1980).

Since, however, experimentally a statistical relationship between the number of faces and the mean radius of this class of grains is found (e.g. Beck 1950, Feltham, 1957, Aboav and Langdon 1969), this relationship can be included into the considerations as an experimental fact, thus reducing the variables again to the grain size, only.

An "ideal" grain structure is mostly assumed to be isotropic i.e. the mean radius of a class of grains is assumed to be independent of the direction. Then it must be assumed that all grain boundary orientations are equally frequent. Hence, the dependence of grain boundary energy and mobility on boundary orientation may be averaged out. It remains only the orientation difference $\Delta g$ (the misorientation) across the boundaries between neighbouring grains.

Under certain statistical assumptions the orientation differences between grain pairs can be expressed by the orientations $g$ of the crystallites. Hence, a statistical theory of grain growth can be formulated with the variables "grain size $r$ " and "grain orientation g." Under these premises a polycrystalline structure is statistically completely described by a distribution function $n(r, g)$ which includes the grain size distribution (independent of orientation) and the texture (independent of grain size). If these parameters are really relevant and sufficient it should be possible to formulate a satisfactory theory using only these parameters.

It can, however, be forseen that the parameters $r$ and $g$ alone will not be completely sufficient to characterize a polycrystalline structure. The growth process will certainly favour low energy boundaries which correspond to certain orientation differences $\Delta g$. Then $\Delta g$ has to be considered additionally as an independent variable. Such a theory must be formulated with a distribution function $n(r, g, \Delta g)$ which comprises grain size distribution, the texture and the misorientation distribution function (or the orientation correlation function).

In the present paper we shall restrict ourselves, however, to the simpler case 
which can be formulated with a distribution function $n(r, g)$ i.e. we allow for a texture in the material but not for orientation correlation.

A theory of this type has been formulated by Abbruzzese and Lücke (1986a, b) and by Bunge and Dahlem $(1986,1987)$. The two theories are similar in their assumptions. Lücke and Abbruzzese assumed the mean curvature of a class of grains as the driving force whereas Bunge and Dahlem considered the balance of grain boundary energy of the surrounding " $a$ " boundaries of a grain pulling "inwards" and the " $b$ " boundaries between two neighbouring grains pulling "outwards." This latter model has the advantage that it allows to take the different orientation mean values of the grain boundary energy for " $a$ " and " $b$ " boundaries into account. In both theories similar differential equations for $n(r, g)$ are derived. Abbruzzese and Lücke solved their equation by numerical integration. To our opinion this is problematic since at $r=0$ the flow rate of the disappearing grains takes on the form $0 \cdot \infty$. Hence, this is a singular point which is very sensitive to the modalities of numerical integration with finite steps of $r$. On the other hand, however, the whole growth process is essentially determined just by those grains disappearing from the structure because their radius converges to zero. Therefore, Bunge and Dahlem used a partly analytical solution (analytical with respect to the variable $r$ ).

Recently Atkinson (1988) published a review on grain growth. Thereby he defined two attributes of "normal" grain growth, 1) a "uniform appearence" i.e. a relatively narrow range of grain sizes and shapes and 2) "scaling" i.e. the growth structure is geometrically similar (in a statistical sense) to the starting structure. Both attributes are, however, only fulfilled in certain idealized cases i.e. in cases where the texture does not change very much. These are especially the completely random case and the case of a sharp single component texture such as the cube texture (as long as one does not consider the sharpening of the texture within the spread range of the cube orientation). For these ideal situations a growth rate law $\bar{r} \sim t^{1 / 2}$ has often been reported. The same growth rate law has also been deduced by several theories (see e.g. Atkinson 1988). On the other hand, also deviating growth rate laws were observed. This was mostly attributed to deviations of the material from the ideal situation e.g. by impurities or particles. Strong deviations were also observed in the case of textures changing during grain growth (Brickenkamp, Lücke 1981; Dahms, Welch, Bunge 1982; Distl, Welch, Bunge 1983; Bunge, Lee, Dahlem 1984; Bunge, Nauer-Gerhardt 1985; Eichelkraut, Lücke, Abbruzzese 1986; Eichelkraut, Abbruzzese, Lücke 1988). In these cases also deviations from the attribute: "uniform appearance," were observed, though much smaller ones than in the case of discontinuous grain growth or secondary recrystallization.

The second attribute: "scaling," may also be violated if one looks into details. True scaling requires a "steady state" grain size distribution curve, the specific form of which is a result of the growth process. This steady state grain size distribution curve has been measured experimentally and it can be deduced from corresponding growth theories. In the most general case, it must be assumed that the shape of this curve, immediately after primary recrystallization, does not necessarily correspond to the steady state form. Hence, transient phenomena will occur in the beginning until a steady state shape of this curve is reached. During this period, deviations from the ideal steady state growth rate law must also be expected. 
On the other hand, "scaling" very often seems to be a rather good approximation. Hence, it may be tempting to compose a general solution of the differential equation of grain growth from several steady state solutions. This line will be followed in the present considerations.

In the steady state, i.e. with a constant form of the grain size distribution curve, an analytical solution of the growth equation can be found which has still a free parameter. Hence, several such solutions with different values of this "shape parameter" can be added in order to obtain a wider class of most general solutions. The case consisting of two steady state components was considered in more detail. Thereby it turned out that always one of the two steady state components finally vanishes. This means, if we start with a grain size distribution curve which deviates from the steady state shape then the growing grain structure automatically converges towards the steady state which is in agreement with many experimental results.

\section{GENERAL DEFINITIONS}

A grain in a polycrystalline material may be characterized by its crystal orientation $g$ defined, for instance, by the Euler angles

$$
g=\left\{\varphi_{1}, \phi, \varphi_{2}\right\}
$$

and by its size which will be specified by the grain radius $r$. In a two-dimensional or three-dimensional model respectively, $r$ is related to the area $A$ or Volume $V$ of the grain

$$
r=\left(\frac{A}{\pi}\right)^{1 / 2} ; \quad r=\left(\frac{3 V}{4 \pi}\right)^{1 / 3}
$$

The number $d N$ of grains of the size $r$ and orientation $g$ in the unit volume of the sample at the time $t$ defines an "orientation-size" distribution function

$$
n(r, g, t)=\frac{d N(r, g, t)}{d r d g}
$$

From this function, the grain size distribution-irrespective of crystal orientation - can be deduced

$$
v(r, t)=\oint n(r, g, t) d g
$$

The mean grain diameter is obtained from $v(r, t)$ by

$$
\bar{r}(t)=\frac{\int_{0}^{\infty} v(r, t) \cdot r \cdot d r}{\int_{0}^{\infty} v(r, t) \cdot d r}
$$

The texture (by volume) is defined by

$$
f(g, t)=\frac{4 \pi}{3} \int_{0}^{\infty} n(r, g, t) r^{3} d r
$$


The total volume of the material is assumed to be unity

$$
V=\oint f(g, t) d g=1
$$

We can also define the texture by number

$$
f^{N}(g, t)=\int_{0}^{\infty} n(r, g, t) d r
$$

Furthermore, we define particular mean values taken over the grains of the orientation $g$, only. The mean radius of these grains is defined by

$$
\tilde{r}(g, t)=\frac{\int_{0}^{\infty} n(r, g, t) r d r}{\int_{0}^{\infty} n(r, g, t) d r}
$$

and the mean volume of $g$-grains is

$$
\tilde{v}(g, t)=\frac{4 \pi}{3} \frac{\int_{0}^{\infty} n(r, g, t) r^{3} d r}{\int_{0}^{\infty} n(r, g, t) d r}
$$

It can also be expressed in terms of the ratio of the two definitions of the texture

$$
\tilde{v}(g, t)=\frac{f(g, t)}{f^{N}(g, t)}
$$

The orientation difference between two neighbouring grains which have the orientations $g_{1}$ and $g_{2}$ is defined by

$$
\Delta g=g_{2} \cdot g_{1}^{-1}
$$

Then the area fraction $d A / A$ of grain boundaries having the "misorientation" $\Delta g$ defines a misorientations distributions function (MODF)

$$
F(\Delta g, t)=\frac{d A(\Delta g, t) / A(t)}{d \Delta g}
$$

This function will be considered separately for the " $a$ "-boundaries surrounding a grain of orientation $g$ (whereby it is assumed that the MODF does not depend on the size $r$ of the grain) and the " $b$ "-boundaries, Figure 1. It is assumed

$$
F^{b}(\Delta g, t)=\oint F^{a}(\Delta g, g, t) \cdot f(g, t) d g
$$

If the neighbouring grain orientations are statistically uncorrelated then the 


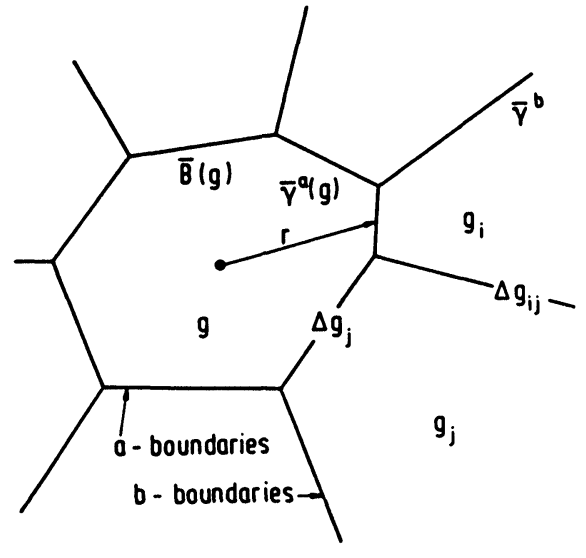

Figure 1 Definition of " $a$ " and " $b$ " boundaries, crystal orientations, misorientations and averaged grain boundary properties.

misorientation functions are completely determined by the texture

$$
\begin{aligned}
& F_{u}^{a}(\Delta g, g, t)=\frac{1}{N^{c}} \sum_{c} f\left(\Delta g \cdot g^{c} \cdot g, t\right) \\
& F_{u}^{b}(\Delta g, t)=\oint f(\Delta g \cdot g, t) \cdot f(g, t) d g
\end{aligned}
$$

where $g^{c}$ are the crystal symmetry rotations.

In the most general case neighbouring grain orientations will, however, be correlated. Then a correlation function $\varphi(\Delta g, t)$ is defined by (Bunge, Weiland 1988)

$$
\varphi(\Delta g, t)=\frac{F(\Delta g, t)}{F_{u}(\Delta g, t)}
$$

(The correlation function must be assumed to be the same for " $a$ " and " $b$ " boundaries). The correlation function $\varphi(\Delta g, t)$ is an independent structural parameter which is not defined by the grain orientation-size function $n(r, g, t)$, i.e. it is not defined by the texture. In the following we shall use a simplified model with

$$
\varphi(\Delta g, t) \equiv 1
$$

The misorientation functions $F^{a}$ and $F^{b}$ define the mean properties of " $a$ " and " $b$ " boundaries, Figure 1, i.e. the mean specific grain boundary energies and the mean mobilities in terms of the misorientation dependence of mobility $B(\Delta g)$ and boundary energy $\gamma(\Delta g)$

$$
\begin{gathered}
\bar{B}^{a}(g, t)=\oint B(\Delta g) \cdot F^{a}(\Delta g, g, t) d \Delta g \\
\bar{\gamma}^{a}(g, t)=\oint \gamma(\Delta g) \cdot F^{a}(\Delta g, g, t) d \Delta g \\
\bar{\gamma}^{b}(t)=\oint \gamma(\Delta g) \cdot F^{b}(\Delta g, t) d \Delta g
\end{gathered}
$$


During grain growth, grains may grow (or shrink). The mean growth rate of the $(r, g)$-grains is given by

$$
\dot{r}=\frac{d r}{d t}=\bar{G}(r, g, t)=\bar{B}^{a}(g, t) \cdot \bar{P}(r, g, t)
$$

where $\bar{P}$ is a driving force given by the variation of the total grain boundary energy $E$ on a virtual change of the grain radius referred to the circumference $u$ of a two-dimensional grain or the surface area $A$ of a three-dimensional grain respectively

$$
\bar{P}=-\frac{1}{u} \cdot \frac{\delta E}{\delta r} ; \quad \bar{P}=-\frac{1}{A} \cdot \frac{\delta E}{\delta r}
$$

The energy $E$ is expressed by the areas of " $a$ " and " $b$ " boundaries, Figure 1, and $\bar{\gamma}^{a}$ and $\bar{\gamma}^{b}$. The grain orientation $g$ remains constant during grain growth. Hence, a flux $\phi$ of grains in the $(r, g)$ space may be defined, Figure 2

$$
\phi(r, g, t)=n(r, g, t) \cdot \bar{G}(r, g, t)
$$

Since no grains disappear except for $r=0$, the distribution function $n$ must obey a continuity equation for $r \neq 0$

$$
\frac{\delta n(r, g, t)}{\delta t}=-\frac{\delta \phi(r, g, t)}{\delta r}
$$
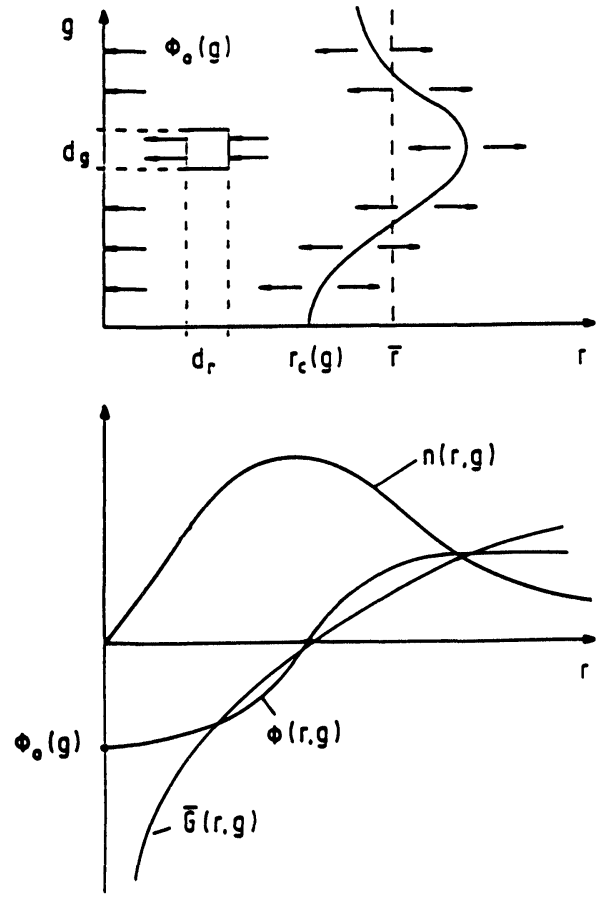

Figure 2 Illustration of the fundamental quantities of grain growth theory. a) The $r-g$-space and flux of grains in $r$-direction, $\phi_{0}(g)$ flux of disappearing grains at $r=0, r_{c}(g)=$ critical radius. b) The grain flux $\phi(r, g)$ is the product of the growth rate $\bar{G}(r, g)$ and the size distribution $n(r, g)$. 
The total loss rate of $g$-grains is determined by the flux at $r=0$

$$
\int_{0}^{\infty} \frac{\delta n(r, g, t)}{\delta t} \cdot d r=-\phi(0, g, t)
$$

Most of the specific growth rate models, including the one used in this paper Eq. (28) postulate an infinite growth rate at $r=0$ (Figure 2). The flux of grains at $r=0$ must, however, be finite. Hence, the grain size distribution curve $n(r, g, t)$ must go to zero for $r \rightarrow 0$. This provides some difficulties if numerical integration with respect to the variable $r$ is used.

The considerations up to here are quite general without taking recourse to a specific model of the polycrystalline structure or to a specific growth rate model.

\section{A SPECIFIC GROWTH RATE MODEL}

A specific growth rate model was considered by Bunge and Dahlem $(1986,1987)$ assuming a two-dimensional grain structure, Figure 3, and the relationship between grain size and number of corners $m$,

$$
m=m_{0}+b \cdot \frac{r}{\bar{r}}
$$

which was first proved by Feltham (1957). This leads to the growth rate law (Bunge, Dahlem 1987)

$$
\bar{G}(r, g, t)=\bar{B}^{*}(g, t)\left[\frac{1}{r_{c}(g, t)}-\frac{1}{r}\right]
$$

with

$$
\begin{aligned}
\bar{B}^{*}(g, t) & =\bar{B}^{a}(g, t) \cdot \bar{\gamma}^{b}(t)\left[\frac{\bar{\gamma}^{a}(g, t)}{\bar{\gamma}^{b}(t)}-\frac{m_{0}}{a}\right] \\
r_{c}(g, t) & =\bar{r}(t) \cdot C(g, t) \\
C(g, t) & =\frac{a}{b}\left[\frac{\bar{\gamma}^{a}(g, t)}{\bar{\gamma}^{b}(t)}-\frac{m_{0}}{a}\right]
\end{aligned}
$$

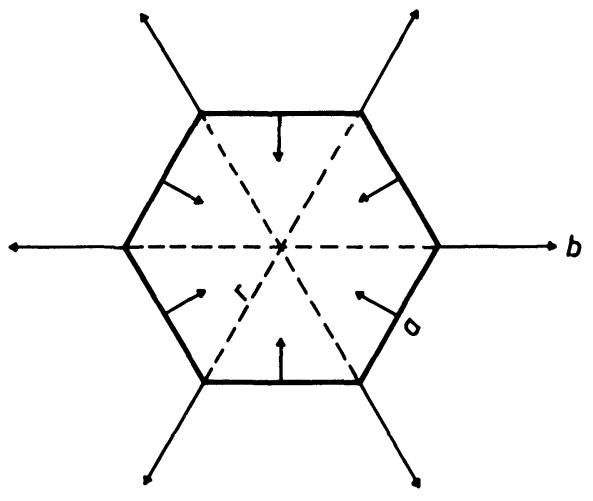

Figure 3 Two-dimensional growth rate model ( $m$-sided regular polygon) " $a$ "-boundaries pulling inwards, " $b$ "-boundaries pulling outwards. 
with the constants

$$
a \approx 2 \pi, \quad b \approx 2.5, \quad m_{0} \approx 3
$$

with Eq. (28), the continuity equation Eq. (25) takes on the form

$$
\frac{\delta n(r, g, t)}{\delta t}=-\bar{B}^{*}(g, t)\left[\frac{n(r, g, t)}{r^{2}}+\left(\frac{1}{C(g, t) \cdot \bar{r}(t)}-\frac{1}{r}\right) \frac{\delta n(r, g, t)}{\delta r}\right]
$$

Then the $t$-dependence of the quantities $\bar{B}^{*}(g, t)$ and $C(g, t)$ comes in by the change of the texture $f(g, t)$ which defines the mean values $\bar{B}^{a}, \bar{\gamma}^{a}$ and $\bar{\gamma}^{b}$ according to Eqs. (19), (20), (21) and Eqs. (15), (16). The values of the parameters $a, b, m_{0}$ in Eq. (32) are slightly model dependent, i.e. they depend on the shapes of the grains and the statistical distribution of the shapes. Particularly, they are slightly different for a simple 2-D or a simple 3-D model. The form of the growth rate expression, Eq. (28), and hence of the continuity equation, Eq. (33), seems, however, to be quite general, at least for a wide class of geometrical models. Hence, the influence of the geometrical model on the final results will be considered later by a slight variation of the numerical values of the "constants" $a$, $b, m_{0}$.

\section{INTEGRATION OF THE CONTINUITY EQUATION}

As long as the texture changes are assumed to be small, the $t$-dependence of the quantities $\bar{B}^{*}$ and $C$ may be neglected. Under these assumptions a steady state solution of Eq. (33) can be obtained. A steady state solution is defined by a constant shape of the grain size distribution curve ("scaling") as is shown in Figure 4

where

$$
n(r, g, t)=A(g, t) \cdot m(g, \rho)
$$

$$
\rho=\frac{r}{\bar{r}(t)}
$$

is the relative grain size. According to Eq. (34) the distribution of the relative grain sizes $m(g, \rho)$ of $g$-grains is constant and only the "amount" $A(g, t)$ of these grains depends on time. The "momenta" of the relative grain size curve are defined by

$$
\bar{m}^{j}(g)=\int_{0}^{\infty} m(g, \rho) \cdot \rho^{j} d \rho
$$

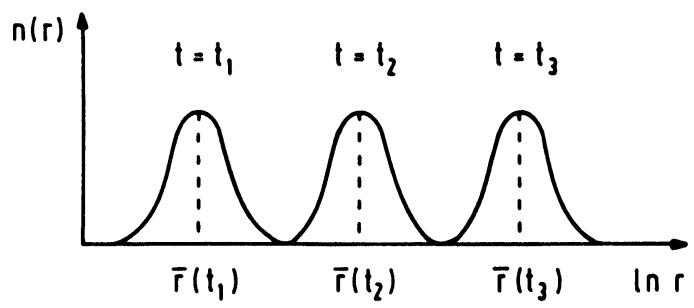

Figure 4 Steady state grain size distribution curve (scaling). 
The function $m(g, \rho)$ may be assumed to be normalized such that

$$
\bar{m}^{3}(g)=1
$$

With these definitions, the mean radius and the mean volume of $g$-grains, Eqs. (9) and (10) take on the simple form

$$
\begin{aligned}
& \tilde{r}(g, t)=\bar{r}(t) \frac{\bar{m}^{1}(g)}{\bar{m}^{0}(g)} \\
& \tilde{v}(g, t)=\frac{4 \pi}{3} \bar{r}^{3}(t) \frac{1}{\bar{m}^{0}(g)}
\end{aligned}
$$

Furthermore, the value $\rho=\lambda(g)$ where $m(g, \rho)$ takes on its maximum is defined by

$$
\left[\frac{\delta m(g, \rho)}{\delta \rho}\right]_{\rho=\lambda(g)}=0
$$

The parameter $\lambda$ defines the shape of the grain size distribution curve as is shown in Figure 5. With the definition of the texture, Eq. (6), the definitions Eqs. (34), (35) and the normalization Eq. (37), the texture function $f(g, t)$ is related to the function $A(g, t)$ defined in Eq. (34)

$$
f(g, t)=\frac{4 \pi}{3} A(g, t) \cdot \bar{r}^{4}(t)
$$

Hence, Eq. (34) can also be written in the form

$$
n(r, g, t)=\frac{3}{4 \pi} \cdot \frac{1}{\bar{r}^{4}(t)} \cdot f(g, t) \cdot m(g, \rho)
$$

Hence, the "amount"-function $A$ is no longer needed. Under these assumptions analytical expressions for the three functions $\bar{r}, f, m$ and hence for the distribution function $n(r, g, t)$ were obtained (Bunge, Dahlem 1986, 1987) which

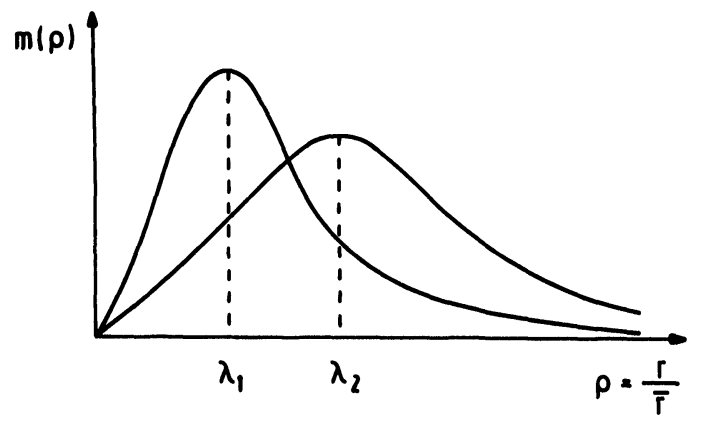

Figure 5 The shape of a grain size distribution curve characterized by the shape parameter $\lambda$, i.e. the most-frequent relative grain size. 
can be written in the form

$$
\begin{gathered}
\bar{r}(t)=\bar{r}_{0}\left[1+\frac{2 K}{\bar{r}_{0}^{2}} \cdot t\right]^{1 / 2} \\
f(g, t)=f(g, 0) \cdot\left[1+\frac{2 K}{\bar{r}_{0}^{2}} \cdot t\right]^{2-\left(\bar{B}^{*}(g) / 2 K \cdot \lambda^{2}(g)\right)} \\
m(g, \rho)=\mu(g) \cdot \rho\left[1-\frac{\rho}{C(g)}+\frac{\rho^{2} K}{\bar{B}^{*}(g)}\right]^{\beta(g)} \\
\cdot \exp \left[\alpha(g) \cdot \operatorname{arctg} \frac{1}{W(g)}\left(\frac{\rho \cdot 2 K}{\bar{B}^{*}(g)}-\frac{1}{C(g)}\right)\right]
\end{gathered}
$$

with

$$
\begin{gathered}
W(g)=\left[\frac{4 K}{\bar{B}^{*}(g)}-\frac{1}{C^{2}(g)}\right]^{1 / 2} \\
\beta(g)=-\frac{1}{2} \cdot\left[1+\frac{\bar{B}^{*}(g)}{\lambda^{2}(g) K}\right] \\
\alpha(g)=\frac{1}{W(g) \cdot C(g)} \cdot\left[1-\frac{\bar{B}^{*}(g)}{\lambda^{2}(g) K}\right] \\
K=\frac{1}{4} \oint f(g, 0) \frac{\bar{B}^{*}(g)}{\lambda^{2}(g)} d g
\end{gathered}
$$

The normalization factor $\mu(g)$ is defined in accordance with Eq. (37). The solution Eqs. (43)-(45) contains the free parameter $\lambda(g)$ which must be chosen in such a way that the total volume $V$, Eq. (7), remains constant and that Eq. (45) is consistent with Eq. (43). These two requirements lead to the side-conditions

$$
\begin{gathered}
\oint f(g, 0)\left[\bar{m}^{1}(g)-\bar{m}^{0}(g)\right] d g=0 \\
\oint f(g, 0)\left[\bar{m}^{1}(g)-\bar{m}^{0}(g)\right] \frac{\bar{B}^{*}(g)}{\lambda^{2}(g)} d g=0
\end{gathered}
$$

which restrict the possible choices of the shape parameters $\lambda(g)$ for the grain size distribution curves of the crystals of the orientations $g$.

Since the quantities $\bar{B}^{*}(g, t)$ and $C(g, t)$ were assumed to be constant, what they really are not because of the texture dependence, the solution given above is only an approximation which is the better valid the shorter the time interval $\Delta t$ in which it is being considered. Hence, the solution has to be repeated with the texture $f(g, \Delta t)$ obtained at the end of the interval $\Delta t$ replacing the starting texture in the next interval. Since the quantity $K$ Eq. (49) depends on the texture (and hence on the time) the mean grain size $\bar{r}(t)$ does then no longer follow the ideal square root law as in the elementary interval $\Delta t$ expressed by Eq. (43). The present approximation is justified as long as the time dependence of $K$ is small compared with the time dependence of $\bar{r}$ expressed by Eq. (43). 


\section{CONSTANT-TEXTURE SOLUTION}

With the particular choice of the shape parameter (critical shape parameter)

$$
\frac{\bar{B}^{*}(g)}{\lambda_{c}^{2}(g)}=\text { const }=4 K, \quad \lambda_{c}(g)=\sqrt{\frac{\bar{B}^{*}(g)}{4 K}}
$$

the exponent in Eq. (44) becomes zero. Hence in this case the texture remains constant.

$$
f(g, t)=f(g, 0)
$$

With Eq. (52), Eq. (49) is identically fulfilled. Furthermore, the two side equations, Eqs. (50) and (51), become identical. They fix the only remaining parameter $K$. Hence, with the choice of $\lambda$ according to Eq. (52), all parameters in Eq. (45) are truly time-independ, i.e. the assumption Eq. (34) is exactly fulfilled and the growth law Eq. (43) is truly parabolic. Hence, with Eq. (52), an exact solution of the growth equation Eq. (33) is obtained. In this solution the texture remains unchanged. With Eq. (52), each texture component has its own particular grain size distribution curve.

$$
\begin{aligned}
m_{c}(g, \rho)= & \mu(g) \cdot \rho\left[1-\frac{\rho}{C(g)}+\frac{\rho^{2} K}{\bar{B}^{*}(g)}\right]^{-5 / 2} \\
& \times \exp \left[\frac{-3}{W(g) \cdot C(g)} \cdot \operatorname{arctg} \frac{1}{W(g)}\left(\frac{\rho \cdot 2 K}{\bar{B}^{*}(g)}-\frac{1}{C(g)}\right)\right]
\end{aligned}
$$

with $W(g)$ according to Eq. (46) and $K$ chosen so that Eq. (50) is fulfilled.

This solution of the growth equation plays a central role for the pseudostationary solutions. It is seen from Eq. (44) that a particular texture component grows or shrinks depending on its shape parameter $\lambda(g)$.

$$
\lambda(g) \begin{array}{ll}
>\lambda_{c}(g) & \text { growth } \\
<\lambda_{c}(g) & \text { shrinkage }
\end{array}
$$

Hence, the critical shape parameter $\lambda_{c}(g)$ decides about growth or shrinkage of a whole texture component similar to the critical radius $r_{c}(g, t)$, Eq. (30), which decides about growth or shrinkage of grains of the size $r$ within this texture component.

\section{DISCRETIZATION OF THE SOLUTION}

The solution Eqs. (42)-(45) is a continuous one with respect to the orientation $g$. We shall now assume that only discrete orientations

$$
g=g_{i} ; \quad i=1,2, \ldots I
$$

occur. Then we obtain a discrete solution in the form

$$
n_{i}(r, t)=\frac{3}{4 \pi} \cdot \frac{1}{\bar{r}^{4}(t)} \cdot f_{i}(t) \cdot m_{i}(\rho)
$$


The momenta of the relative grain size distribution curve of the orientation component $i$ are defined according to Eq. (36) by

$$
\bar{m}_{i}^{j}=\int_{0}^{\infty} m_{i}(\rho) \cdot \rho^{j} d \rho
$$

The normalization of the relative grain size distribution is given by

$$
\bar{m}_{i}^{3}=1
$$

and the shape parameter $\lambda_{i}$ follows from

$$
\left[\frac{d m_{i}(\rho)}{d \rho}\right]_{\rho=\lambda_{i}}=0
$$

The solution Eqs. (43), (44), (45) takes on the form

$$
\begin{gathered}
\bar{r}(t)=\bar{r}_{0}\left[1+\frac{2 K}{\bar{r}_{0}^{2}} \cdot t\right]^{1 / 2} \\
f_{i}(t)=f_{i}(0) \cdot\left[1+\frac{2 K}{\bar{r}_{0}^{2}} \cdot t\right]^{2-\left(\bar{B}_{i}^{*} / 2 K \cdot \lambda_{i}^{2}\right)} \\
m_{i}(\rho)=\mu_{i} \cdot \rho\left[1-\frac{\rho}{C_{i}}+\frac{\rho^{2} K}{\bar{B}_{i}^{*}}\right]^{\beta_{i}} \cdot \exp \left[\alpha_{i} \cdot \operatorname{arctg} \frac{1}{W_{i}}\left(\frac{\rho \cdot 2 K}{\bar{B}_{i}^{*}}-\frac{1}{C_{i}}\right)\right]
\end{gathered}
$$

with the abbreviations

$$
\begin{gathered}
W_{i}=\left[\frac{4 K}{\bar{B}_{i}^{*}}-\frac{1}{C_{i}^{2}}\right]^{1 / 2} \\
\beta_{i}=-\frac{1}{2} \cdot\left[1+\frac{\bar{B}_{i}^{*}}{\lambda_{i}^{2} K}\right] \\
\alpha_{i}=\frac{1}{W_{i} \cdot C_{i}} \cdot\left[1-\frac{\bar{B}_{i}^{*}}{\lambda_{i}^{2} K}\right] \\
K=\frac{1}{4} \sum_{i}^{I} f_{i} \frac{\bar{B}_{i}^{*}}{\lambda_{i}^{2}}
\end{gathered}
$$

And the side conditions take on the form

$$
\begin{gathered}
\sum_{i}^{I} f_{i}(0)\left[\bar{m}_{i}^{1}-\bar{m}_{i}^{0}\right]=0 \\
\sum_{i}^{I} f_{i}(0)\left[\bar{m}_{i}^{1}-\bar{m}_{i}^{0}\right] \cdot \frac{\bar{B}_{i}^{*}}{\lambda_{i}^{2}}=0
\end{gathered}
$$

Since there are $I$ orientations $g_{i}$ for each of which a shape parameter $\lambda_{i}$ is defined but only two side conditions among them, the restriction imposed by the side 
conditions is the less severe the higher the number of orientations. Also the Eqs. (19), (20), (21) have to be written in the discrete form.

$$
\begin{gathered}
\bar{B}_{i}^{a}=\sum_{j}^{I} B_{i j} \cdot f_{j} \\
\bar{\gamma}_{i}^{a}=\sum_{j}^{I} \gamma_{i j} \cdot f_{j} \\
\bar{\gamma}^{b}=\sum_{1, j}^{I} \gamma_{i j} \cdot f_{i} \cdot f_{j}
\end{gathered}
$$

and finally the quantities $\bar{B}_{i}^{*}$ and $C_{i}$ are defined

$$
\begin{gathered}
\bar{B}_{i}^{*}=\bar{B}_{i}^{a} \cdot \bar{\gamma}^{b} \cdot\left[\frac{\bar{\gamma}_{i}^{a}}{\bar{\gamma}^{b}}-\frac{m_{0}}{a}\right] \\
C_{i}=\frac{a}{b} \cdot\left[\frac{\bar{\gamma}_{i}^{a}}{\bar{\gamma}^{b}}-\frac{m_{0}}{a}\right]
\end{gathered}
$$

The discrete solution will be the basis for the numerical calculations which will be considered later on.

\section{NON-STEADY STATE SOLUTION}

The discrete solution given above is a pseudo-steady state one which is expressed in Eq. (57). The relative grain size distribution $m_{i}(\rho)$ of a crystal orientation $g_{i}$ remains constant throughout the integration interval $\Delta t$. Only the amount of this component i.e. the texture $f_{i}(t)$ changes during the interval. This allows an easy generalization to a non-steady state solution. We assume that some of the orientations $g_{i}$ are identical e.g.

$$
g_{i_{1}}=g_{i_{2}}=g_{i_{3}}=\cdots g_{k}
$$

Then the grain size distribution of grains of orientation $g_{k}$ is no longer "pseudo-steady state" during the integration interval. The size distribution of these grains is given by

$$
\bar{n}_{k}(r, t)=\frac{3}{4 \pi} \cdot \frac{1}{\bar{r}^{4}(t)} \sum_{x=1}^{\kappa} f_{i_{x}}(t) \cdot m_{i_{x}}(\rho)
$$

This is a function which changes its shape in the interval $\Delta t$ since the $f_{i_{x}}(t)$ are variable with $t$. A schematic example showing two orientations, each of which consists of two steady state components is shown in Figure 6. If we keep the definition of the function $f_{i}(t)$ as being the volume fraction of the individual pseudo-steady state component $i$ then the texture i.e. the volume fraction of the orientation $g_{k}$ must be re-defined. It is

$$
\bar{f}_{k}(t)=\sum_{x=1}^{\kappa} f_{i_{x}}(t)
$$

In this case, the quantities $\bar{B}^{a}, \bar{\gamma}^{a}, \bar{\gamma}^{b}$ Eqs. (70), (71), (72) are to be defined with 


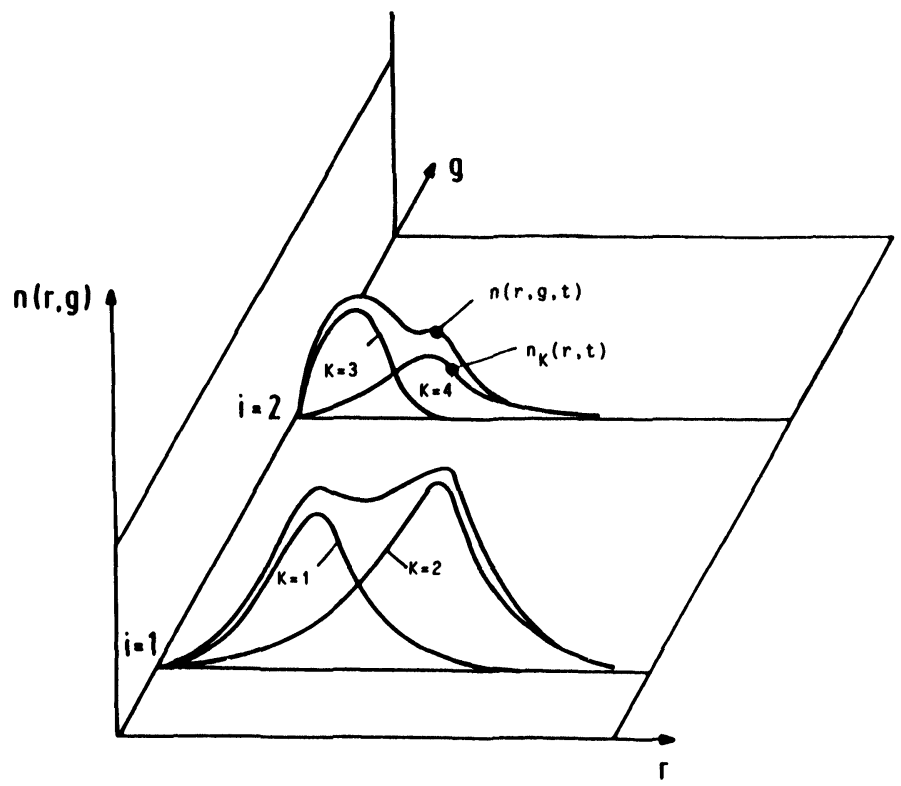

Figure 6 The orientation-size distribution function for the case of two orientations, each of which consists of two pseudo-steady state size distribution components.

the "true" texture function $\bar{f}_{k}(t)$ instead of the former definition with $f_{i}$. Hence, these quantities are the same for all "steady state" components $i_{x}$ belonging to the same "orientation component" $k$. Also the quantities $\bar{B}^{*}$ and $C$ depend only on the orientation component $k$. This case will be considered in more detail in another paper.

\section{ONE STEADY STATE COMPONENT}

If there is only one steady state component

$$
I=1
$$

the equations Eqs. (57), (61)-(66) take on the simple form

$$
\begin{gathered}
n(r, t)=\frac{3}{4 \pi} \cdot \frac{1}{\bar{r}^{4}(t)} \cdot m(\rho) \\
r(t)=\bar{r}_{0}\left[1+\frac{\bar{B}^{*}}{2 \lambda^{2} \bar{r}_{0}^{2}} \cdot t\right]^{1 / 2} \\
m(\rho)=\mu \rho\left[1-\frac{\rho}{C}+\frac{\rho^{2}}{4 \lambda^{2}}\right]^{-5 / 2} \cdot \exp \left[-\frac{3}{W C} \cdot \operatorname{arctg} \frac{1}{W}\left(\frac{\rho}{2 \lambda^{2}}-\frac{1}{C}\right)\right] \\
W=\left[\frac{1}{\lambda^{2}}-\frac{1}{C^{2}}\right]^{1 / 2}
\end{gathered}
$$


and the side conditions Eqs. (68), (69) take on the common form

$$
\bar{m}^{1}=\bar{m}^{0}
$$

which can also be written

$$
\int_{0}^{\infty} m(\rho)(1-\rho) d \rho=0
$$

This is a truely analytical solution which is truely "steady state" in the sense that the relative grain size distribution curve $m(\rho)$ is truely (not only in an interval $\Delta t$ ) constant. This case has been treated numerically by Bunge and Dahlem (1987).

\section{TWO STEADY STATE COMPONENTS}

In this case the equations Eqs. (67)-(69) read

$$
\begin{gathered}
K=\frac{1}{4}\left[\frac{f_{1}(0) \bar{B}_{1}^{*}}{\lambda_{1}^{2}}+\frac{f_{2}(0) \bar{B}_{2}^{*}}{\lambda_{2}^{2}}\right] \\
f_{1}(0)\left[\bar{m}_{1}^{1}-\bar{m}_{1}^{0}\right]+f_{2}(0)\left[\bar{m}_{2}^{1}-\bar{m}_{2}^{0}\right]=0 \\
\frac{f_{1}(0) \bar{B}_{2}^{*}}{\lambda_{1}^{2}}\left[\bar{m}_{1}^{1}-\bar{m}_{1}^{0}\right]+\frac{f_{2}(0) \bar{B}_{2}^{*}}{\lambda_{2}^{2}}\left[\bar{m}_{2}^{1}-\bar{m}_{2}^{0}\right]=0
\end{gathered}
$$

with

$$
f_{1}(0)+f_{2}(0)=1
$$

Eqs. (86), (87) can be fulfilled in two ways which define two branches of solutions:

Branch 1 (constant-texture solution). If we put according to Eq. (52)

$$
\frac{\bar{B}_{1}^{*}}{\lambda_{1}^{2}}=\frac{\bar{B}_{2}^{*}}{\lambda_{2}^{2}}=4 K
$$

then Eq. (87) is identical with Eq. (86). According to Eq. (53) this is a solution without texture change.

Branch 2 (solution with texture change). Eqs. (86), (87) can also be fulfilled simultaneously by putting:

$$
\bar{m}_{1}^{1}=\bar{m}_{1}^{0} ; \quad \bar{m}_{2}^{1}=\bar{m}_{2}^{0}
$$

This is a solution with texture changes. The solutions of branch 1 and 2 together provide the complete spectrum of solutions of Eqs. (86), (87). With two components present, only two types of grain boundaries occur, i.e. boundaries $i=j$ between grains of the same component, these are low angle boundaries (ideally they are no boundaries at all since it is $\Delta g=0)$ and $(1,2)$ boundaries between grains of the two components. We introduce the ratios of the boundary energies and mobilities of the two types of boundaries.

$$
\frac{B_{12}}{B_{i i}}=x ; \quad \frac{\gamma_{12}}{\gamma_{i i}}=y
$$


then Eqs. (70)-(72) read

$$
\begin{gathered}
\bar{B}_{i}^{a}=B_{i i}\left[f_{i}+x \cdot f_{j}\right] \\
\bar{\gamma}_{i}^{a}=\gamma_{i i}\left[f_{i}+y \cdot f_{j}\right] \\
\bar{\gamma}^{b}=\gamma_{i i}\left[\left(f_{1}^{2}+f_{2}^{2}+y \cdot f_{1} f_{2}\right]\right.
\end{gathered}
$$

The parameters $\bar{B}_{i}^{*}$ and $C_{i}$ are then expressed by

$$
\begin{gathered}
\bar{B}_{i}^{*}=B_{i i} \cdot \gamma_{i i}\left[f_{i}+x f_{j}\right]\left[f_{i}+y f_{j}-\frac{m_{0}}{a}\left(f_{1}^{2}+f_{2}^{2}\right)-\frac{m_{0}}{a} 2 y f_{1} \cdot f_{2}\right] \\
C_{i}=\frac{a}{b}\left[\frac{f_{i}+y f_{j}}{f_{1}^{2}+f_{2}^{2}+2 y f_{1} \cdot f_{2}}-\frac{m_{0}}{a}\right]
\end{gathered}
$$

where $f_{1}$ and $f_{2}$ are related to each other by Eq. (88). It is seen that the absolute values of grain boundary energy and mobility enter these relationships only in the form of the product $B_{i i} \cdot \gamma_{i i}$. According to Eqs. (29) and (67) also the parameter $K$ is proportional to this value. Hence, the ratio $\bar{B}_{i}^{*} / K$ is independent of $B_{i i} \cdot \gamma_{i i}$. According to Eqs. (64)-(66) the same holds for the parameters $W_{i}, \beta_{i}, \alpha_{i}$. Finally it also holds for $m_{i}(\rho)$ according to Eq. (63) and also to the side conditions Eqs. (68), (69) from which the parameters $\lambda_{i}$ are to be determined. The shapes of the grain size distributions are thus independent of the absolute values of mobility and energy. They depend only on the misorientation dependence of these quantities expressed by the parameters $x$ and $y$. The factor $B_{i i} \cdot \gamma_{i i}$ enters only Eqs. (61) and (62) via $K$ as a factor of the time $t$. Hence, the absolute value $B_{i i} \cdot \gamma_{i i}$, so to speak, changes only the time scale of grain growth but not the shapes of the distribution functions and the texture development. If we put in Eq. (86)

$$
f_{1}(0)=f_{2}(0)=\frac{1}{2}
$$

then it follows from Eq. (87)

$$
\frac{\bar{B}_{1}^{*}}{\lambda_{1}^{2}}=\frac{\bar{B}_{2}^{*}}{\lambda_{2}^{2}}
$$

Hence this is a solution without texture change. Furthermore, it follows from Eqs. (70), (71), (73), (74)

$$
\bar{B}_{1}^{*}=\bar{B}_{2}^{*}, \quad C_{1}=C_{2}
$$

and with Eq. (98) it also follows

$$
\lambda_{1}=\lambda_{2}
$$

Hence, the two texture components have also the same grain size distribution curve which is identical with that of the one-component case. The assumption, Eq. (97), thus leads to the one-component case.

If the grain boundary energy and mobility are independent of the orientation difference then we have to put in Eq. (91)

$$
x=y=1
$$


With this assumption we obtain from Eqs. (92)-(95)

$$
\bar{B}_{1}^{*}=\bar{B}_{2}^{*}
$$

and Eq. (86), (87) have the only solution

$$
\lambda_{1}=\lambda_{2}
$$

Hence, in this case no texture change occurs and the grain size distribution curves of both texture components are identical to those of the one-component case.

Finally, Eq. (102) also holds if the orientations $g_{1}$ and $g_{2}$ are the same. Because of Eq. (103) the two corresponding grain size distribution curves then are the same. This means, a non-steady state solution as assumed in Eq. (76) does not exist in the two-component case.

If we consider more than two steady state components which have the same orientation $g_{k}$ according to Eq. (75) then the equivalent to Eq. (102) also holds. However, the argument which lead from Eq. (102) to Eq. (103) does not hold in this case. Hence, with three or more steady state components a non-steady state solution can be obtained even with one orientation component. This case will be discussed elsewhere.

\section{NUMERICAL RESULTS}

\section{One component case}

The simplest case is that of one steady state component which may be a sharp single-orientation texture or the case of a completely random material. In this case it is according to Eqs. (29), (31)

$$
\begin{gathered}
\bar{B}^{*}=\bar{B} \cdot \bar{\gamma} \cdot\left[1-\frac{m_{0}}{a}\right]=\bar{B} \cdot \bar{\gamma} \cdot 0.523 \\
C=\frac{a}{b}\left[1-\frac{m_{0}}{a}\right]=1.313
\end{gathered}
$$

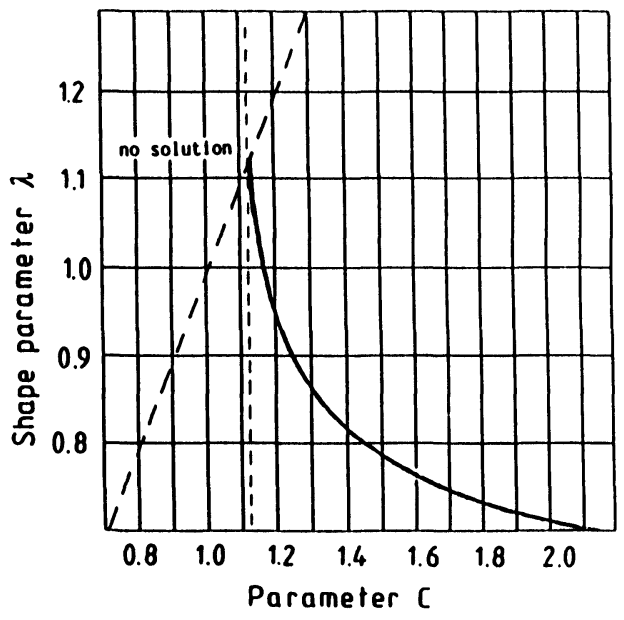

Figure 7 Relationship between shape parameter $\lambda$ and the constant $C$ defining the critical radius in the case of one steady state component. 
Table 1 Values of the parameter $C$ and corresponding values of the shape parameter $\lambda$

\begin{tabular}{lll}
\hline$C$ & $\lambda$ & Comment \\
\hline$<1.13$ & - & no solution \\
1.13 & 1.1 & \\
1.15 & 1.05 & \\
1.313 & 0.857 & present model \\
1.5 & 0.79 & \\
2.0 & 0.71 & \\
\hline
\end{tabular}

The value of $C$ is fixed in this case by the values of the parameters $a, b, m_{0}$. It was, however, mentioned that the values of these latter parameters must be considered as being slightly model-dependent. Hence, the parameter $C$ is considered here as a variable. The parameter $\lambda$ determines the shape of the grain size distribution curve. It is the relative grain size for which this curve takes on its maximum. The condition Eq. (84) establishes a relationship between $\lambda$ and $C$ which can be calculated numerically using Eqs. (81), (82). This relationship is shown in Figure 7. (It is to be mentioned that Figure 7 was published earlier, Bunge, Dahlem 1987 with a wrong scale on the $\lambda$-axis). It is seen that there is no solution for $C<1.13$. The boundary $C=\lambda$ is due to the parameter $W$, Eq. (83), which must be real. Some corresponding values of $C$ and $\lambda$ are given also in Table 1. The present model leads to the value $C=1.313$. The corresponding relative grain size distribution curve is shown in Figure 8. It is not a log-normal curve as can be seen from Figure 8b. Grain size distribution curves for a range of $C$-values are shown in Figure 9. With increasing $C$-value the distribution contains an increasing amount of grains larger than twice the mean grain size $\bar{r}$. According to Eq. (81) the growth rate depends on the shape parameter $\lambda(C)$. This is shown in Figure 10 where the growth curves are expressed in relative scales $r / r_{0}$ and ${ }_{2}^{1} \vec{B}^{*} \cdot \bar{r}_{0}^{-2} t$.

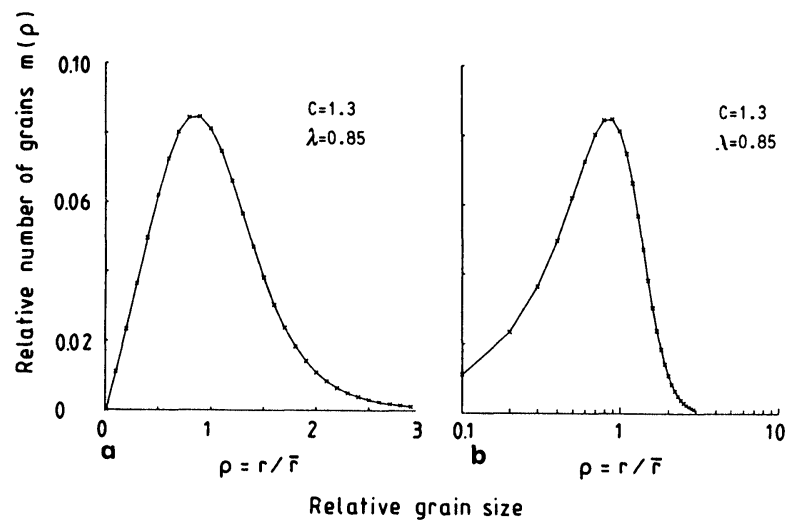

Figure 8 Relative grain size distribution curve according to the present model for one steady state component (sharp single orientation texture or random distribution). a) linear scale. b) logarithmic scale. 

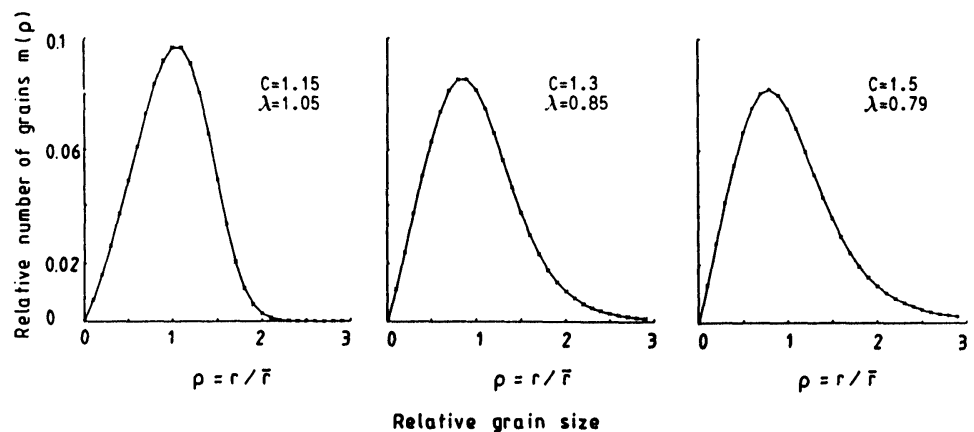

Figure 9 Relative grain size distribution curve for some $C$-values.

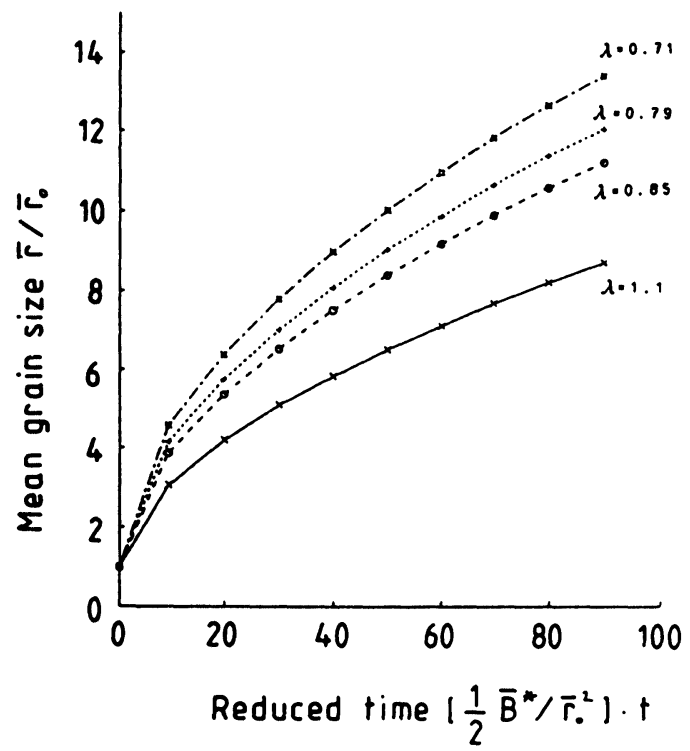

Figure 10 Grain growth curves for various values of the parameter $\lambda$.

\section{Two-component case}

In this case the solution of the side equations Eqs. (86), (87) for the shape parameters $\lambda_{1}, \lambda_{2}$ depends only on the parameters $x, y$, Eq. (91) which describe the $\Delta g$-dependence of grain boundary energy and mobility and on the texture which is in this case described by the volume fraction $f_{1}$ of crystals in the orientation $g_{1}$. The shape parameters do not depend on the absolute values of boundary energy and mobility nor on the mean initial grain size. Hence, a complete survey over this case is obtained by choosing a representative set of points in the $(x-y)$ plane as is shown in Figure 11. As was shown in Eqs. (101)-(103) the case $x=y=1$ is equivalent to the one-component case. The points on the line $x=1$ represent the influence of anisotropic grain boundary energy alone whereas $y=1$ represents the influence of anisotropic mobility only. 


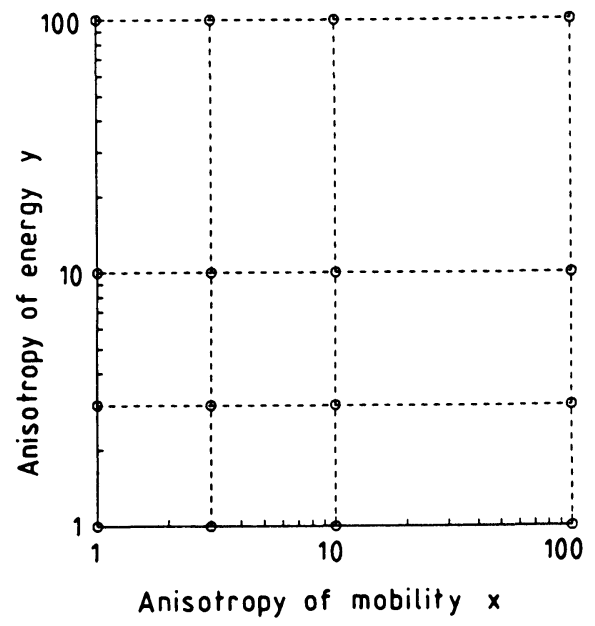

Figure 11 Values of the anisotropy parameters $x$ (mobility) and $y$ (energy) for which model calculations were carried out.

The shape parameters. At first, the shape parameters $\lambda_{1}, \lambda_{2}$ have to be determined such that the two side equations Eqs. (86) and (87) are simultaneously fulfilled. In the constant-texture solution (branch 1 ) the ratio $\lambda_{2} / \lambda_{1}$ is constant according to Eq. (89). The ratio $\bar{B}_{1}^{*} / \bar{B}_{2}^{*}$ and hence $\lambda_{2} / \lambda_{1}$ depends on the texture described by $f_{1}$. Hence, the value of the expression Eq. (86) was calculated numerically for $\lambda_{2} / \lambda_{1}=$ const. and its zero point was determined by interpolation. An example of this is shown in Figure 12a.

The solution with texture change (branch 2 ) is defined by Eq. (90). Hence, the quantities $\bar{m}_{i}^{j}$ Eq. (58) were calculated numerically, using Eq. (63), as a function of $\lambda_{1}, \lambda_{2}$. The solution is then obtained by interpolation as is seen in an example in Figure 12b.

With the anisotropy parameters $x$ and $y$ fixed, the solution still depends on the texture described by $f_{1}$. Figure 13 gives an example of possible $\lambda_{1}, \lambda_{2}$ values for different textures as indicated along the curves. The solution must be symmetrical with respect to the line $\lambda_{2}=\lambda_{1}$. Exchange of $f_{1}$ and $f_{2}$ leads to the exchange of $\lambda_{2}$ and $\lambda_{1}$. Furthermore, both curves must pass through the point $\lambda_{1}=\lambda_{2}=0.857$, which describes the one-component state as was explained in Eq. (97) to (103).

A survey of solutions depending on the values $x, y$ of the grain boundary anisotropy parameters is contained in Figure 14. In this figure the values $\lambda_{i}$ are plotted as a function of $f_{i}$ (Two corresponding values of $\lambda_{1}$ and $\lambda_{2}$ are thus obtained from Figure 14 by corresponding values of $f_{1}$ and $f_{2}=1-f_{1}$ ). In each partial diagram, either $x$ or $y$ is kept constant and the other one of these parameters varies through the relevant range which was shown in Figure 11.

The solutions $\lambda_{1}, \lambda_{2}$ can also be represented in the $\lambda_{1}, \lambda_{2}$-field as is shown in Figure 15. The partial diagrams of this figure correspond to the same values of $x$ or $y$ as those of Figure 14. Hence, Figure 14 and Figure 15 contain a survey of possible values $\lambda_{1}, \lambda_{2}$ satisfying the two side conditions Eqs. (86), (87) for various values $x, y$ of anisotropy of grain boundary energy and mobility. It is seen, that the influence of both anisotropies on the solution is quite different. 
(a)

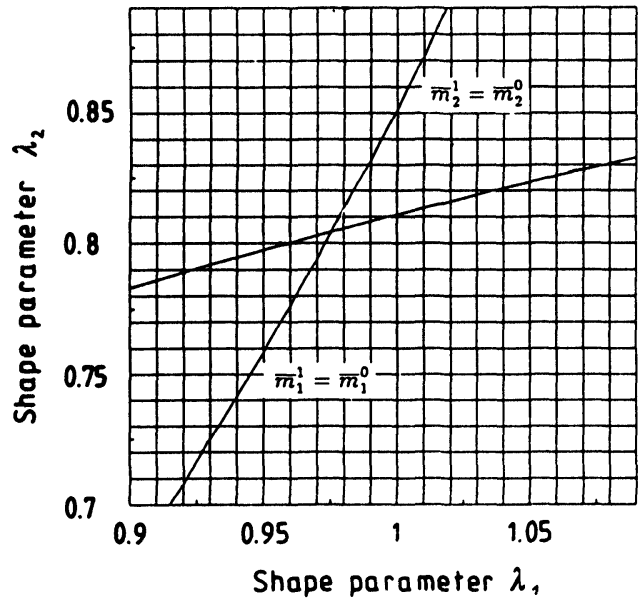

(b)

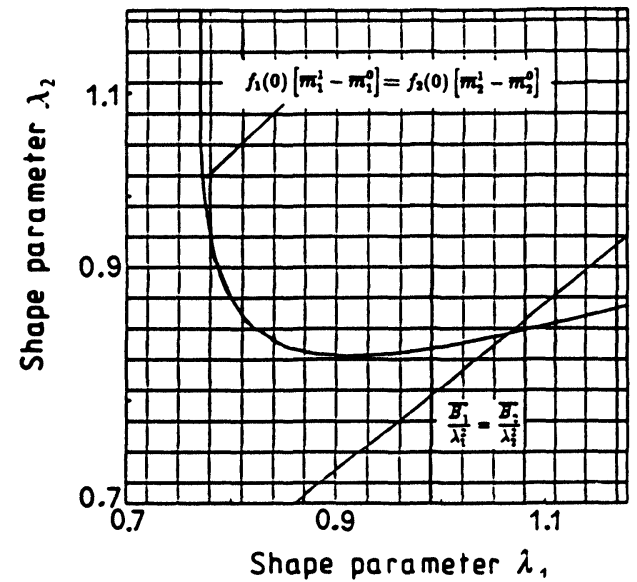

Figure 12 (b) Numerical solution of Eq. (90) for $x=3, y=10, f_{1}=0.45$. Branch 1. (a) Numerical solution of Eq. (86) for $x=3, y=$ $10, f_{1}=0.7$. Branch 2 .

The grain size distribution. The shape parameters $\lambda_{i}$ determine the shape of the grain size distribution curves according to Eq. (63) with the abbreviations Eqs. (64)-(67). A survey of the various grain size distribution curves is given in Figures 16, 17, 18. In Figure 16 the variation of these curves with the anisotropy parameters $x, y$ is shown. Thereby the texture i.e. $f_{1}$ is kept constant (In branch 1 , $f_{1}=0.45$ was chosen and in branch 2 it was $f_{1}=0.25$ ). For better comparison of both curves in each diagram, the curves are not normalized according to Eq. (59). Instead, $\bar{m}_{i}^{o}=1$ was chosen. Hence, the curves in Figure 16 are normalized to the same number of crystallites of orientation 1 and 2 rather than to the same total volume. Figure 17 shows the influence of the texture $f_{1}$ on the shape of the grain size distribution. Finally, Figure 18 contains some examples of partial grain size distribution curves and their addition to the total grain size distribution normalized in terms of volume fractions of grains of orientations 1 and 2. 


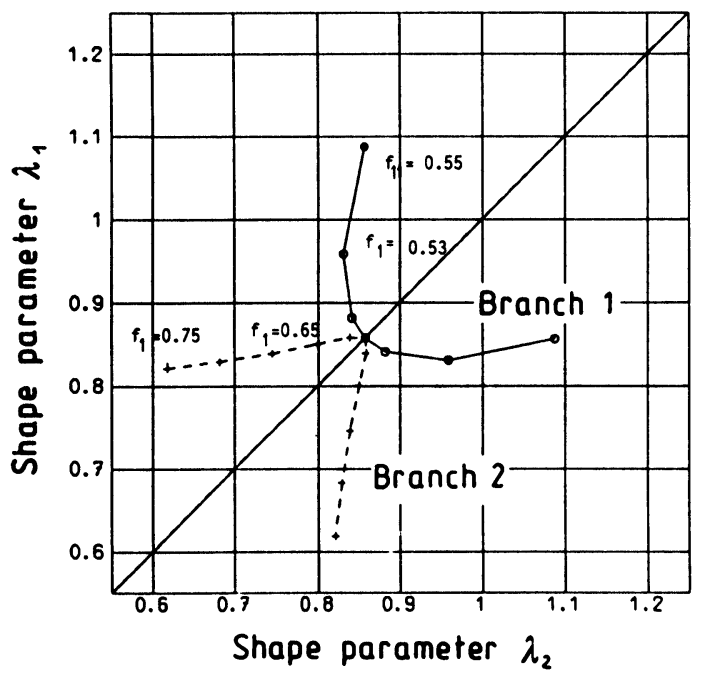

Figure 13 Possible values of shape parameters according to branch 1 and 2 for $x=10, y=10$.

The growth kinetics. The growth kinetics is described by Eqs. (61), (62) which are both valid within a certain time interval in which the quantities $K$ as well as $\bar{B}_{i}^{*}$ and $\lambda_{i}$ can be assumed to be constant. These quantities are truly constant for the solutions of branch 1. In this branch, the texture doesn't change. Hence, the kinetics is completely described, in this case, by Eq. (61) which may then be written

$$
\frac{\bar{r}(t)}{\bar{r}_{0}}=\left[1+\frac{2 K}{\bar{r}_{0}^{2}} \cdot t\right]^{1 / 2}
$$

The growth law is thus truly parabolic and the growth kinetics is defined by the parameter

$$
\frac{2 K}{\bar{r}_{0}^{2}}=\frac{\bar{B}^{*}}{2 \lambda^{2} \bar{r}_{0}^{2}}=\frac{B_{i i} \gamma_{i i}}{2 \bar{r}_{0}^{2}} \cdot \frac{u\left(f_{1}, x, y\right)}{\lambda_{1}^{2}}
$$

where $u$ is an expression given in Eq. (95). The shape parameter $\lambda_{1}$ is determined when $f_{1}, x$ and $y$ are given. The first factor in Eq. (107) is trivial. It contains the absolute values of boundary energy and mobility and the initial grain size. The second factor contains the anisotropy and texture-dependence. Its values for certain cases are given in Table 2 .

\section{CONCLUSIONS}

In the present grain growth model it is assumed that the growth rate of a crystallite depends only on crystal orientation $g$ and grain size $r$. The particular form of the growth rate expression Eq. (28) is based on the driving force obtained 
Branch 1 (constant texture)
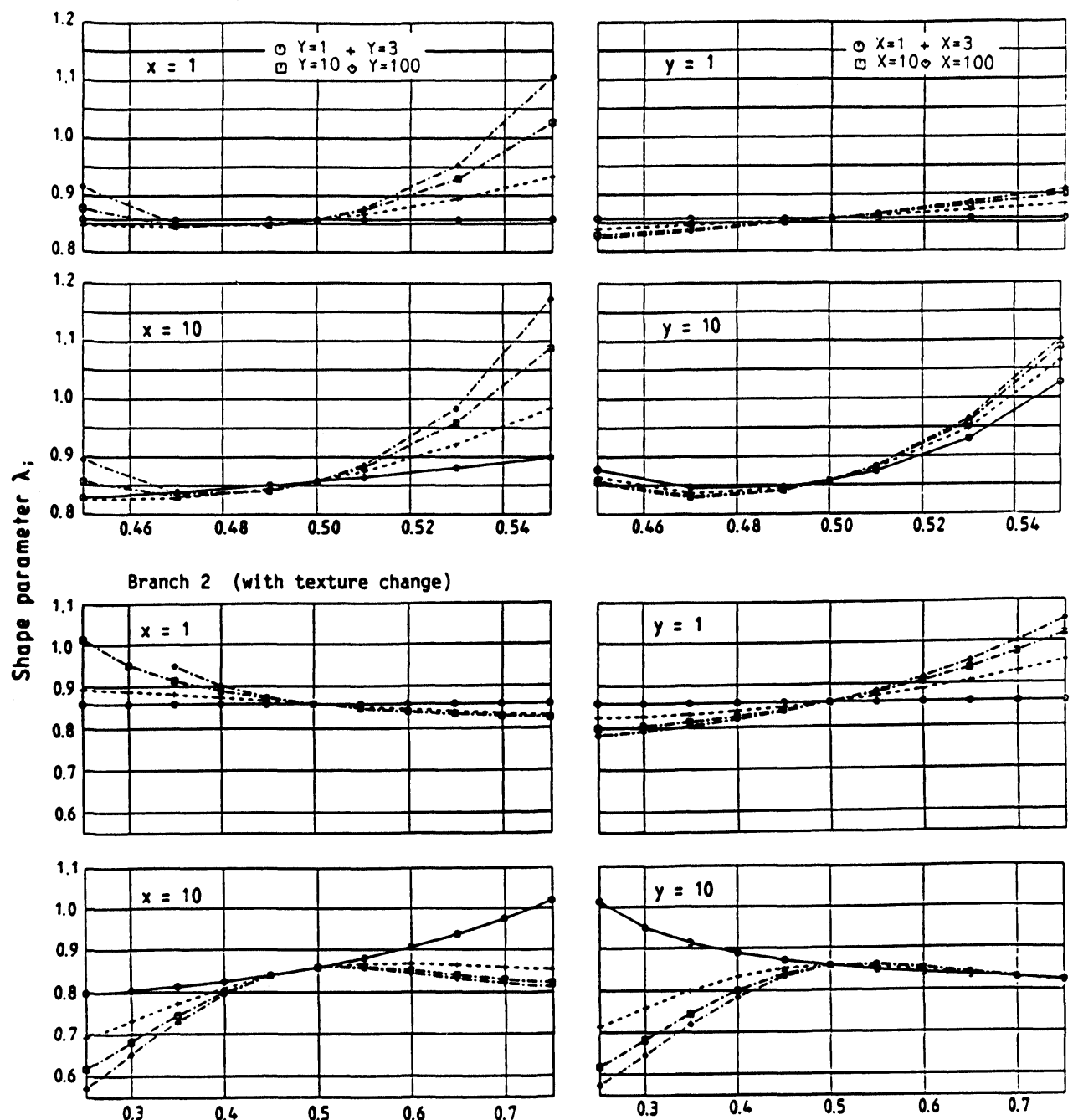

Texture component $f_{i}$

Figure 14 The shape parameter $\lambda_{i}$ as a function of the texture component $f_{i}$. The meaning of the symbols is indicated in the uppermost diagrams.

as the balance between grain boundary forces pulling "inwards" and "outwards" as illustrated in Figure 3. Both kinds of boundaries have different distributions of orientation difference and hence different mean energies depending on the texture of the material. The model leads to a differential equation Eq. (33), the integrals of which represent the number of grains having orientation $g$ and radius $r$ at the time $t$. Under the assumption of a steady state grain size distribution (scaling) for each orientation $g$ this equation can be analytically integrated. 
This assumption is exactly fulfilled in the "one-component" case which holds for single-component sharp textures as well as for completely random structures. The assumption is also exactly fulfilled in the multi-component case (deliberate texture) if each grain orientation $g$ has its own particular grain size distribution curve $m_{c}(g, \rho)$ fixed by the shape parameter $\lambda_{c}(g)$ according to Eqs. (52) and (54). In both these cases the texture remains constant and the mean grain size follows exactly a parabolic law.

In the most general case, the assumption of "scaling" for each orientation $g$ is not strictly fulfilled. Nevertheless, there is a wide class of orientation-size distributions for which it is a good approximation. Also these cases can be treated with the help of the analytical solution, the validity of which is then restricted to a

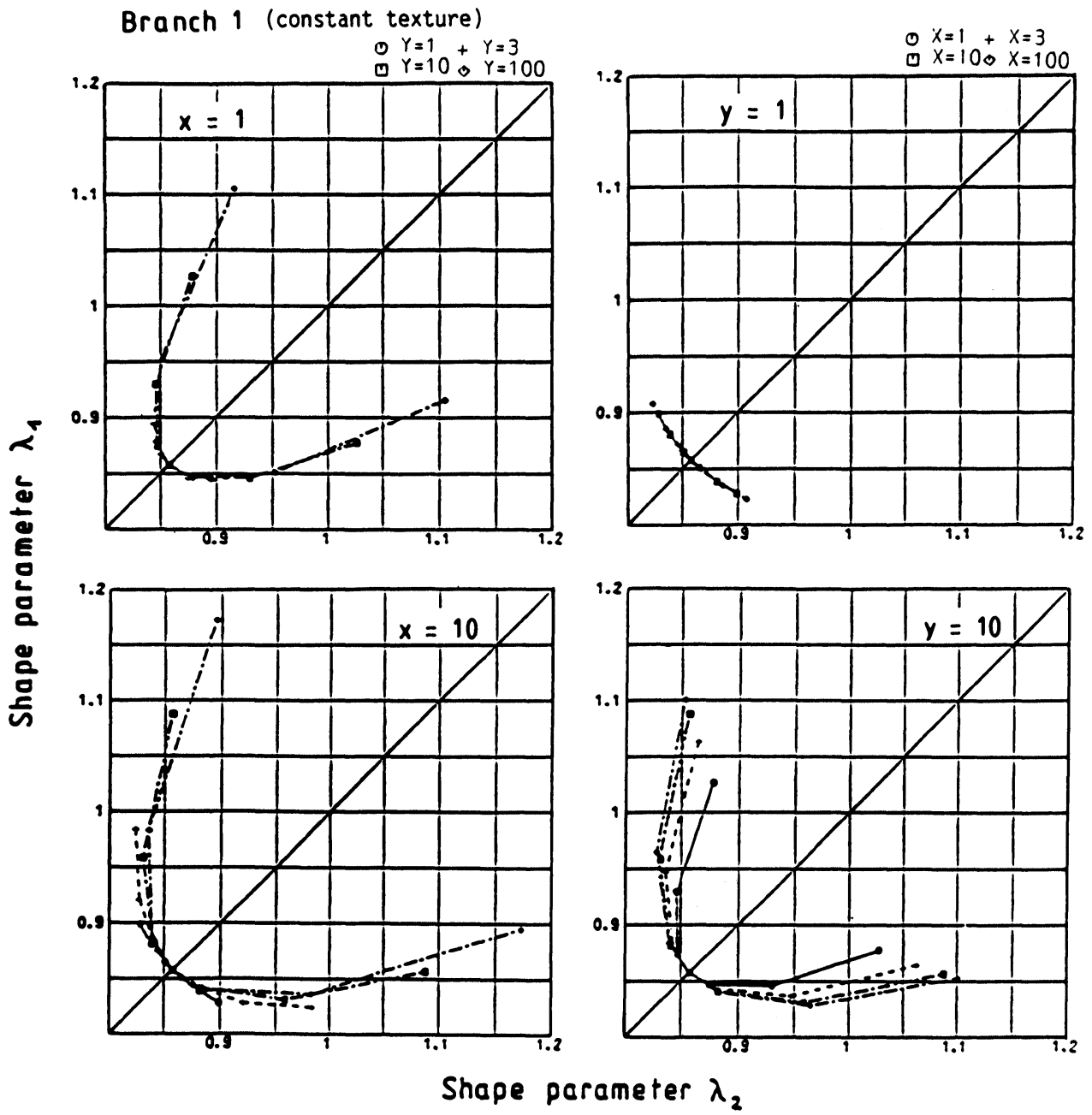

Figure 15 Possible values of shape parameters $\lambda_{1}$ and $\lambda_{2}$, (branch 1). 
Branch 2 (with texture change)

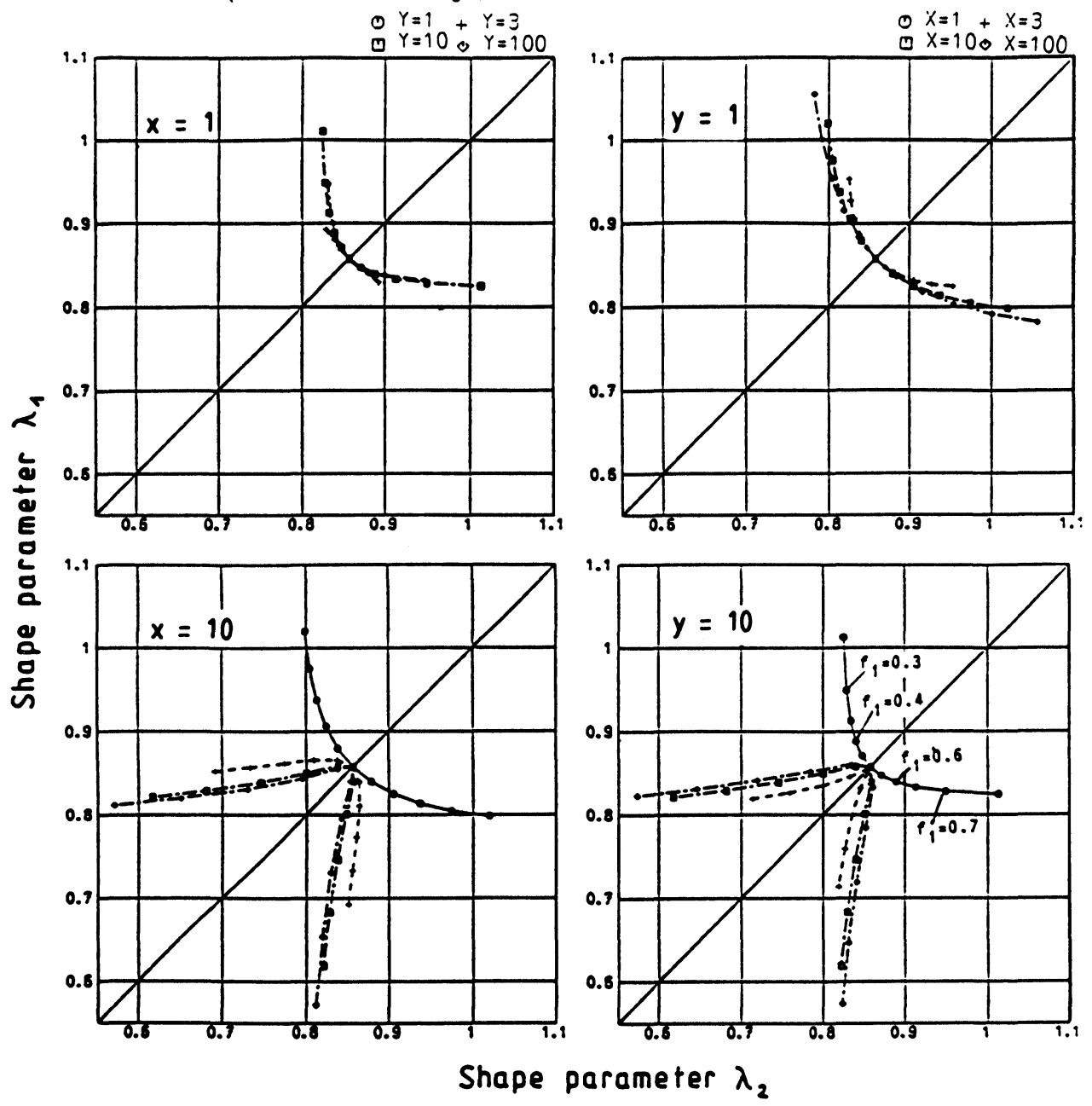

Figure 15 (Continued) Possible values of shape parameters $\lambda_{1}$ and $\lambda_{2}$, (branch 2).

certain time interval $\Delta t$. After that a new solution has to be obtained with constants calculated with the new texture. This approximation is justified when the texture changes are slowly compared with the increase of grain size.

Numerical calculations have been carried out for the one-component case (consisting of one orientation $g$ or random orientation) and for the twocomponent case. In this latter case, only two types of grain boundaries occur, i.e. small angle boundaries between grains of the same component and large angle boundaries between grains of the two orientations. It has been shown that in this case the growth behaviour is determined by the ratio $x$ of energies and $y$ of mobilities of the two types of boundaries. The absolute values of energy and 
mobility have only an influence on the time-scale of grain growth. The anisotropies of grain boundary energy (parameter $x$ ) and mobility (parameter $y$ ) have quite different influence on the growth behaviour.

More general, non-steady state solutions can be "composed" of pseudo-steady state solutions in such a way that several pseudo-steady state orientation-size components are allowed to have the same orientation $g$ but different size distribution curves. It was also shown that in this case at least three steady state components are needed. (In the present paper, however, numerical calculations were restricted to the two-component case.) The numerical results show that the grain growth behaviour (growth or shrinkage) of a particular texture component $g$ depends very sensitively on details of its particular grain size distribution curve. In order to come near to realistic situations, multi-component cases must be considered which have an extremely increasing number of degrees of freedom in their orientation-size distribution $n(g, r)$. The decomposition of this curve into a finite number of pseudo-steady state components, however, provides the chance to systematically analyse these influences.

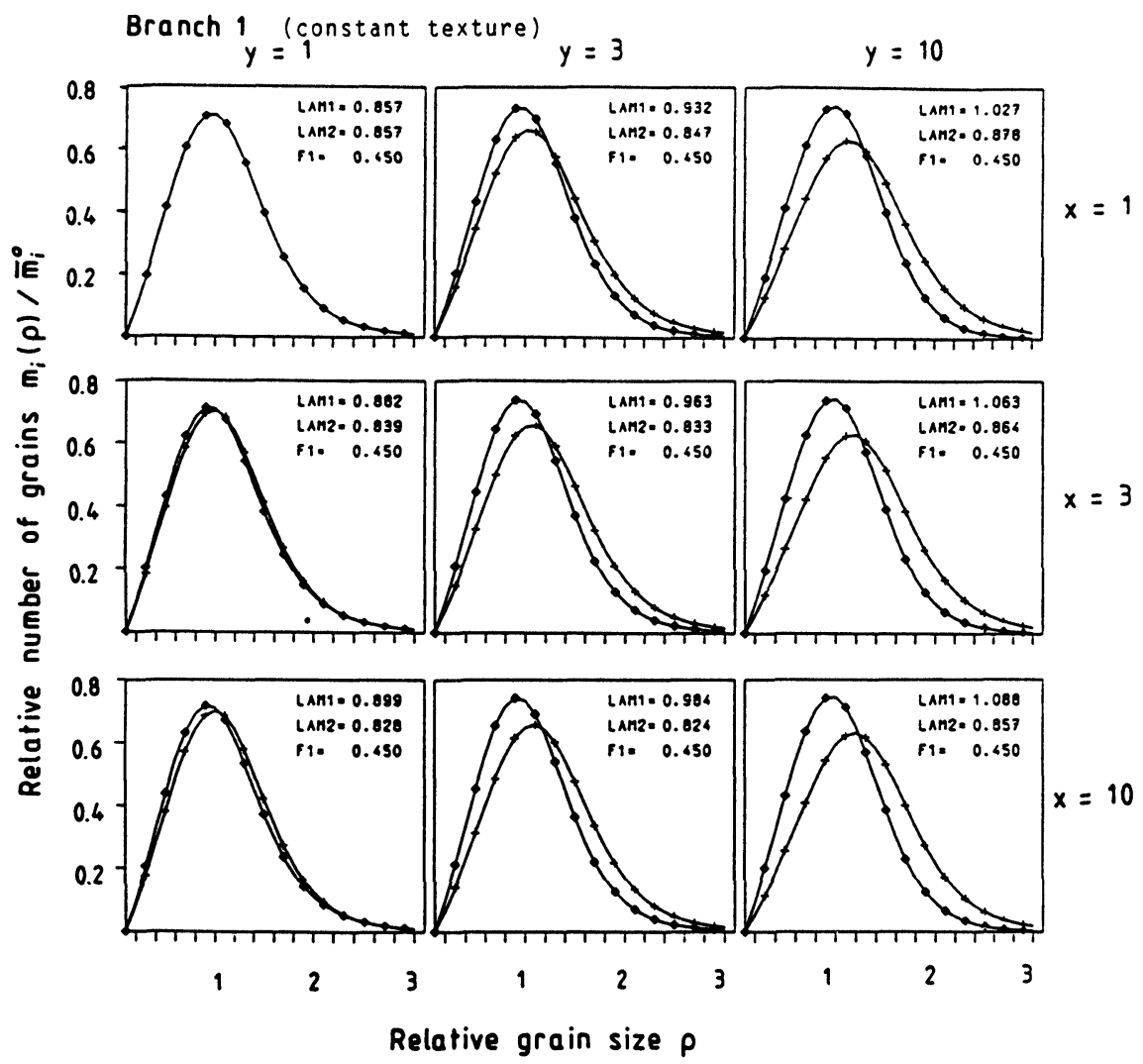

Figure 16 Relative gain size distribution curves $m_{i}(\rho) / m_{i}^{0}$ (normalized to equal numbers of grains 1 and 2). Branch $1 f_{1}=0.45$. 


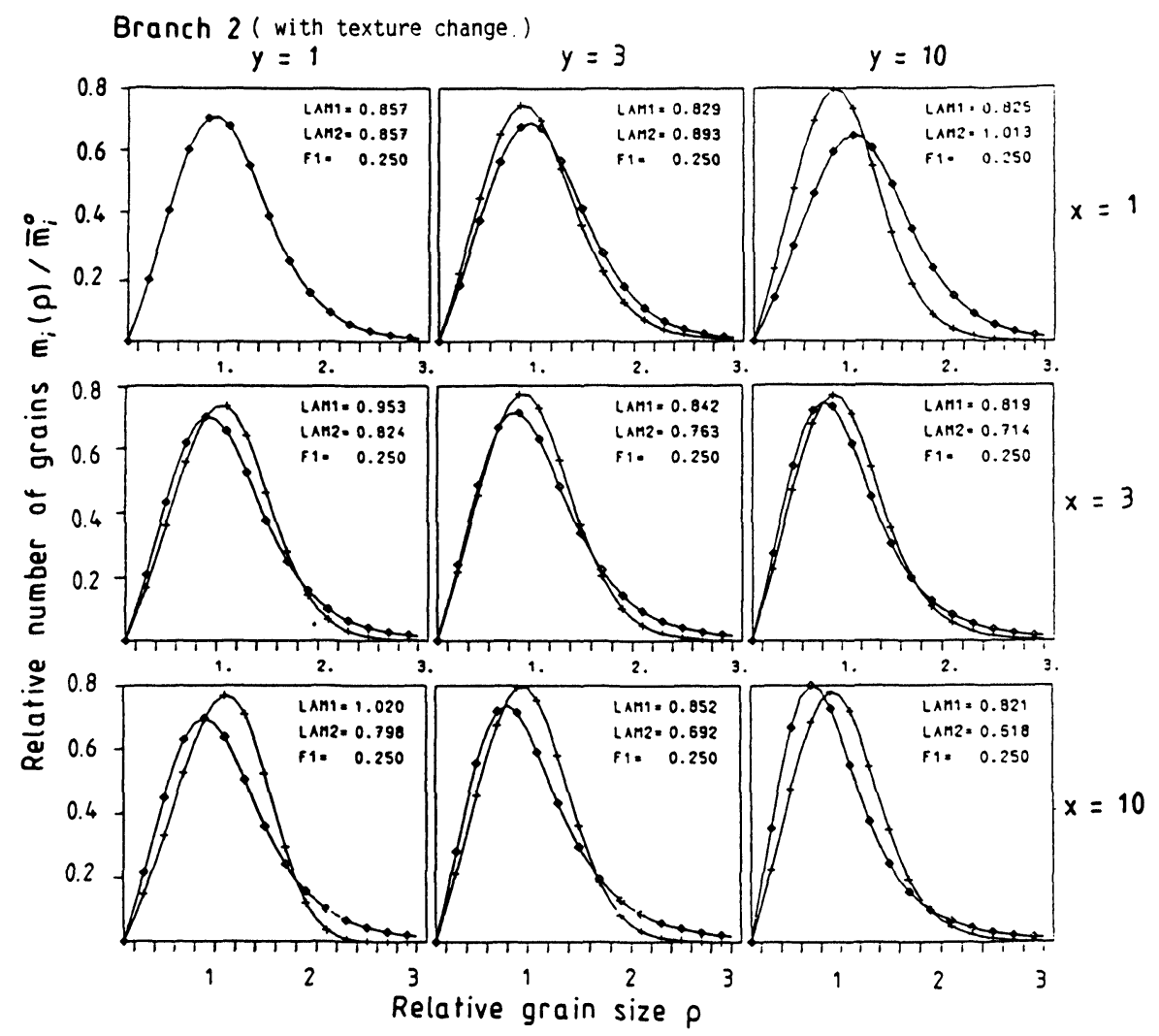

Figure 16 (Continued) Branch $2 f_{1}=0.25$.

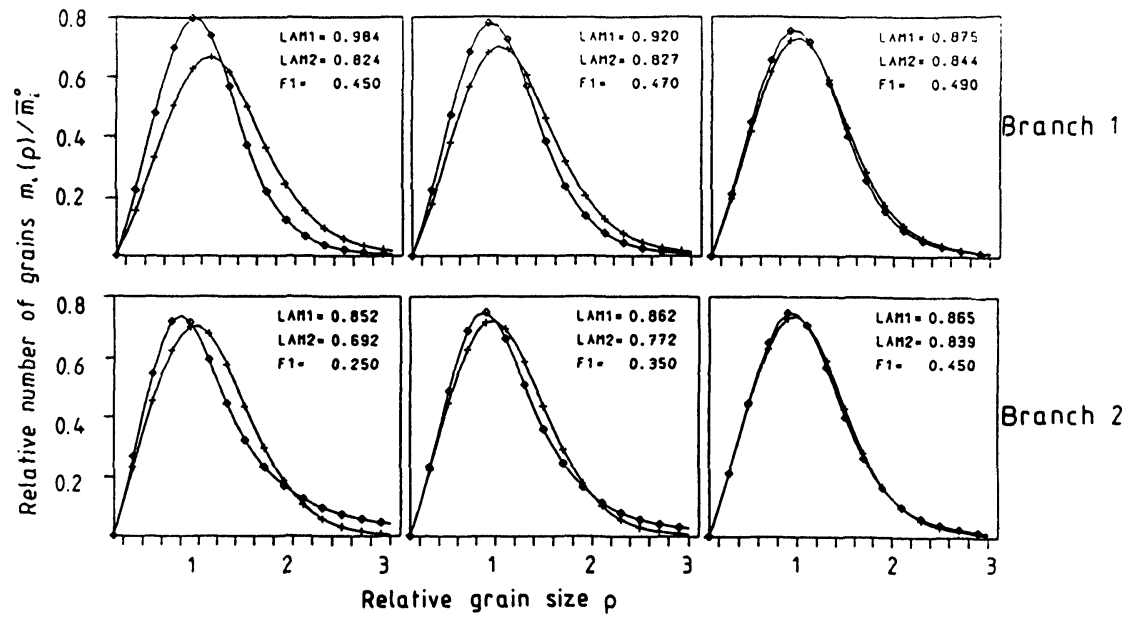

Figure 17 Relative grain size distribution curves $m_{i}(\rho) / m_{i}^{0}$ (normalized to equal numbers of grains 1 and 2) for $x=10, y=3$ and for different textures $f_{1}$. 


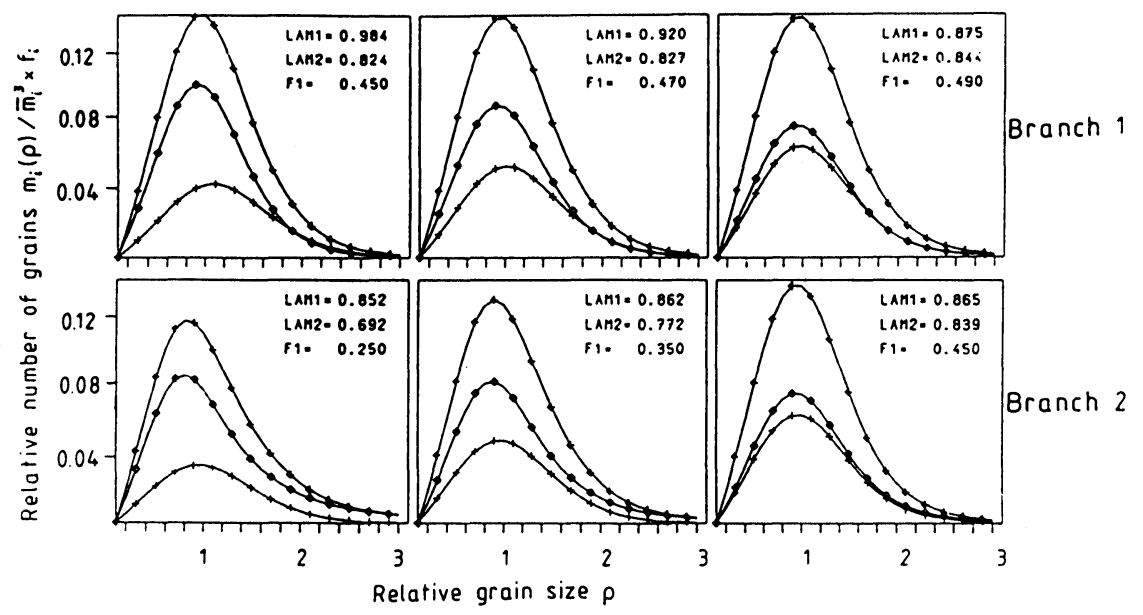

Figure 18 Relative grain size distribution curves $m_{i}(\rho) \cdot f_{i}$ showing size distribution and amount of grains $g_{1}$ and $g_{2}$.

Table 2 Some values of the function $u$ defined in Eq. (107)

\begin{tabular}{rrrrrr}
\hline & \multicolumn{3}{c}{$f_{1}=0.2$} & \multicolumn{3}{c}{$f_{2}=0.8$} \\
\hline$x$ & \multicolumn{1}{c}{$y$} & $u(f, x, y)$ & $x$ & \multicolumn{1}{c}{$y$} & $u(f, x, y)$ \\
\hline 3 & 10 & 17 & 3 & 10 & 1 \\
10 & 1 & 4 & 10 & 1 & 1 \\
10 & 10 & 52 & 10 & 10 & 3 \\
100 & 1 & 42 & 100 & 1 & 11 \\
100 & 10 & 509 & 100 & 10 & 20 \\
10 & 3 & 15 & 10 & 3 & 2 \\
1 & 10 & 6 & 1 & 10 & 1 \\
1 & 100 & 65 & 1 & 100 & 5 \\
10 & 100 & 530 & 10 & 100 & 15 \\
\hline
\end{tabular}

\section{Acknowledgement}

The authors gratefully acknowledge financial support for this project by Deutsche Forschungsgemeinschaft.

\section{References}

Abbruzzese, G. and Lücke, K. (1989). Statistical Theory of Grain Growth Including Texture and Drag Effects and its Application to Texture Controlled Grain Growth. In: Annealing Processes, Recovery and Grain Growth. Proc. 7-th Intern. Symp. Risø. Ed. N. Hansen, D. Juul-Jensen, T. Leffers and B. Ralph, 1-14.

Abbruzzese, G. and Lücke, K. (1986). A Theory of Texture Controlled Grain Growth. Acta Met. 34, 905-914.

Aboav, D. A. and Langdon, T. G. (1969). The Shape of Grains in a Polycrystal. Metallography, 2, 171-178.

Andersen, M. P. (1986). Simulation of Grain Growth in Two and Three Dimensions. In: Annealing Processes, Recovery and Grain Growth. Proc. 7-th Intern. Symp. Risø. Ed. N. Hansen, D. Juul-Jensen, T. Leffers and B. Ralph, 15-34. 
Atkinson, H. V. (1988). Theories of Normal Grain Growth in Pure Single Phase Systems. Acta Met. 36, 469-491.

Beck, P. A. (1954). Annealing of Cold Worked Metals. Phil. Mag. Suppl. 3, 245-324.

Brickenkamp, W. and Lücke, K. (1981). Texture Development During Grain Growth of Rolled and Annealed $\alpha$-Brass Sheets. Proc., 6-th Intern. Conf. on Textures of Materials, ICOTOM-6, Tokyo, 570-580.

Bunge, H. J. and Dahlem, E. (1987) Steady State Grain Growth in Textured Materials. In: Theoretical Methods of Texture Analysis. Ed. H. J. Bunge. DGM Informationsgesellschaft, Oberursel, 301-324.

Bunge, H. J., Lee, H. P. and Dahlem, E. (1984). Growth Selection and Texture Changes During Continuous Grain Growth. Proc. 7-th Intern. Conf. on Textures of Materials, ICOTOM-7, Nordwijkerhout, 217-220.

Bunge, H. J. and Dahlem, E. (1986). Texture Changes and Growth Anomalies During Continuous Grain Growth. In: Annealing Processes, Recovery and Grain Growth. Proc. 7-th Intern. Symp. Risø. Ed. N. Hansen, D. Juul-Jensen, T. Leffers and B. Ralph, 255-260.

Bunge, H. J. and Weiland, H. (1988). Orientation Correlation in Grain and Phase Boundaries. Textures and Microstructures, 7, 231-263.

Bunge, H. J. and Nauer-Gerhardt, C. U. (1985). Orientation Dependent Grain Growth in Titanium. In: Titanium Science and Technology. Ed. G. Lutjering, K. Zwicker and W. Bunk, DGM Informationsgesellschaft, Oberursel, Vol. 3, 1713-1719.

Bunge, H. J. and Dahlem-Klein, E. (1988). Model Calculations of Grain Growth in Textured Materials. Proc. 8-th Intern. Conf. on Textures of Materials, ICOTOM-8, Santa Fe, 705-710.

Cotteril, P. and Mould, P. R. (1976). Recrystallization and Grain Growth in Metals. Surrey University Press, 266-300.

Dahms, M., Welch, P. I. and Bunge, H. J. (1982). Texture Development in AlMn1 During Continuous Grain Growth. Scripta Met. 16, 827-830.

Distl, J. S., Welch, P. I. and Bunge, H. J. (1983) Anomalous Grain Size Distributions During Continuous Grain Growth. Scripta Met. 17, 975-978.

Eichelkraut, H., Abbruzzese, G. and Lücke, K. (1988). Theory of Texture Controlled Grain Growth. II Numerical and Analytical Treatment of Grain Growth in the Presence of Two Components. Acta Met. 36, 56-68.

Eichelkraut, H., Lücke, K. and Abbruzzese, C. (1986). Texture Controlled Grain Growth in $\mathrm{Cu}-\mathrm{Zn}$ and Al-Mg Alloys. In: Annealing Processes, Recovery and Grain Growth. Proc. 7-th Intern. Symp. Risø. Ed. N. Hansen, D. Juul-Jensen, T. Leffers and B. Ralph, 303-308.

Feltham, P. (1957). Grain Growth in Metals. Acta Met. 5, 97-105.

Grest, G. S., Anderson, M. P. and Srolowitz, D. J. (1980). Computer Simulation of Microstructural Dynamics. In: Computer Simulation of Microstructural Evolution. Ed. D. J. Srolowitz. The Metallurgical Society of AIME, 13-20.

Hillert, M. (1965). On the Theory of Normal and Abnormal Grain Growth. Acta Met. 13, 227-238.

Kurtz, S. K. and Carpey, F. M. A. (1989). Microstructure and Normal Grain Growth in Metals and Ceramics. Part I Theory. J. Appl. Phys. 51, 5725-5744.

Novikov, V. Yu. (1976). The Structure Effect on Selective Growth in Recrystallization. Texture 2, $35-44$.

Novikov, V. Yu. (1979). On Computer Simulation of Texture Development in Grain Growth. Acta Met. 27, 1461-1466. 